\title{
Enhancement of Apx Toxin Production in Actinobacillus pleuropneumoniae Serotypes 1, 2, and 5 by Optimizing Culture Conditions
}

\author{
Hoai Thu Dao', Van Tan Do ${ }^{1}$, Quang Lam Truong ${ }^{2}$, and Tae-Wook Hahn ${ }^{1,3 *}$ \\ ${ }^{1}$ College of Veterinary Medicine and Institute of Veterinary Science, Kangwon National University, Chuncheon \\ 24341, Republic of Korea \\ ${ }^{2}$ Key Laboratory of Veterinary Biotechnology, Faculty of Veterinary Medicine, Vietnam National University of \\ Agriculture, Hanoi, Vietnam \\ ${ }^{3}$ Innovac Co., Kangwon National University, 1 Kangwondaehak-gil, Chuncheon 24341, Republic of Korea
}

Actinobacillus pleuropneumoniae (APP) is a causative agent of porcine pleuropneumonia. Therefore, the development of an effective vaccine for APP is necessary. Here, we optimized the culture medium and conditions to enhance the production yields of Apx toxins in APP serotype 1, 2, and 5 cultures. The use of Mycoplasma Broth Base (PPLO) medium improved both the quantity and quality of the harvested Apx toxins compared with Columbia Broth medium. Calcium chloride $\left(\mathrm{CaCl}_{2}\right)$ was first demonstrated as a stimulation factor for the production of Apx toxins in APP serotype 2 cultures. Cultivation of APP serotype 2 in PPLO medium supplemented with $10 \mu \mathrm{g} / \mathrm{ml}$ of nicotinamide adenine dinucleotide (NAD) and $20 \mathrm{mM} \mathrm{CaCl}{ }_{2}$ yielded the highest levels of Apx toxins. These findings suggest that the optimization of the culture medium and conditions increases the concentration of Apx toxins in the supernatants of APP serotype 1, 2, and 5 cultures and may be applied for the development of vaccines against APP infection.

Keywords: Actinobacillus pleuropneumoniae, Apx toxins, production yields

Received: December 25, 2019 Accepted: March 24, 2020

First published online: March 27, 2020

*Corresponding author Phone: +82-33-2508671 Fax: +82-33-2595625 E-mail: twhahn@kangwon.ac.kr

pISSN 1017-7825 elSSN 1738-8872

Copyright $(2020$ by The Korean Society for Microbiology and Biotechnology

\section{Introduction}

Actinobacillus pleuropneumoniae (APP) is a causative agent of porcine pleuropneumonia, which can cause sudden death in pigs in peracute form, leading to significant economic loss within the worldwide swine industry [1]. Vaccination is one of the most promising approaches to control and prevent porcine pleuropneumonia in pigs $[2,3]$. Currently, the main commercial vaccines are bacterin vaccines, subunit vaccines, and toxoid vaccines [1]. However, 18 serotypes of APP have been identified [4], which contributes to the difficulty in combating the disease effectively.

The main virulence factors of APP include the outer membrane, capsule, repeats-in-toxin toxins, proteases, lipopolysaccharides, adhesins, and transferrin-binding proteins [5]. The virulence of APP is highly variable and depends on the serotype, which is closely correlated with the type of Apx exotoxins secreted [6]. Four types of Apx exotoxins have been identified and exhibit different functions and virulence [1,7]. The ApxI toxin (105 kDa) is strongly hemolytic and strongly cytotoxic; the ApxII toxin $(103 \mathrm{kDa})$ is weakly hemolytic and moderately cytotoxic, while the ApxIII toxin $(120 \mathrm{kDa})$ is not hemolytic but strongly cytotoxic. ApxI, II, and III are considered as the most important virulence factors of APP and can be produced and secreted into culture supernatants. ApxIV $(200 \mathrm{kDa})$ is present in all serovars of APP in natural infection [1].

Apx toxins play important roles in helping APP avoid the host clearance mechanisms, thus inducing lesions, and are highly immunogenic [8]. Therefore, Apx toxins are considered as the most important components and are used widely in the current commercial vaccines [1]. A vaccine comprising ApxI, II, and III and the $42 \mathrm{kDa}$ outermembrane protein showed good results in decreasing lethality and pathology in pigs against a virulent APP challenge [9]. Although several reports have provided methods of production of Apx toxins from APPs, there is a need for approaches that afford improvement of the production yields of these toxins. In this study, we optimized the culture medium and conditions to increase the production yield of Apx toxins from APP serotypes 1,2, and 5. 
Table 1. Primers used in this study.

\begin{tabular}{|c|c|c|c|c|}
\hline Primer & Sequence $\left(5^{\prime} \rightarrow 3^{\prime}\right)$ & Target & PCR product (bp) & Reference \\
\hline \multicolumn{5}{|c|}{ Primers used for identifying APP } \\
\hline P1 & TGGCACTGACGGTGATGA & apxIVA & 442 & {$[10]$} \\
\hline P2 & GGCCATCGACTCAACCAT & & & \\
\hline P3 & GGGGACGTAACTCGGTGATT & apxIVA & 378 & \\
\hline $\mathrm{P} 4$ & GCTCACCAACGTTTGCTCAT & & & \\
\hline \multicolumn{5}{|c|}{ Primers used for serotyping APP } \\
\hline P5 & GGGCAAGCCTCTGCTCGTAA & APP1 & 754 & {$[12]$} \\
\hline P6 & GAAAGAACCAAGCTCCTGCAAT & & & \\
\hline P7 & CGCAGCCGGACAAAAACAAATACACG & APP2 & 1724 & {$[11]$} \\
\hline P8 & CACCCCATGAATCGACTGATTGCCAT & & & \\
\hline P9 & TTTATCACTATCACCGTCCACACCT & APP5 & 1107 & [13] \\
\hline P10 & CATTCGGGTCTTGTGGCTACTAAA & & & \\
\hline
\end{tabular}

\section{Materials and Methods}

\section{Strains and Growth Conditions}

The APP serotypes 1,2, and 5 were isolated from the lungs of pigs with contagious pleuropneumonia in Korea and were stored at $-70^{\circ} \mathrm{C}$. The primers used in this study for identifying and serotyping APP are listed in Table 1 . These primers were synthesized by Cosmogenetech (Korea). The APP strains were identified via nested PCR using primer pairs P1, P2, P3, and P4 (Table 1), as described previously [10]. Subsequently, multiplex PCR in a total of $20 \mu \mathrm{l}$ was used for serotyping [11-13]. Briefly, $5 \mu \mathrm{l}$ of the suspended colony was mixed into the PCR premix containing $2 \times$ TOPsimple DyeMIX-Tenuto (Enzynomics, Korea) and 10 pmol of each primer (P5, P6, P7, P8, $\mathrm{P} 9$, and P10) (Table 1). The thermal cycling conditions included an initial denaturation at $95^{\circ} \mathrm{C}$ for $5 \mathrm{~min}$, followed by 35 cycles of $95^{\circ} \mathrm{C}$ for $1 \mathrm{~min}, 55^{\circ} \mathrm{C}$ for $1 \mathrm{~min}, 72^{\circ} \mathrm{C}$ for $1 \mathrm{~min} 50 \mathrm{~s}$; and a final extension at $72^{\circ} \mathrm{C}$ for $10 \mathrm{~min}[11]$.

The APP isolates were grown in nutrient medium supplemented with $10 \mu \mathrm{g} / \mathrm{ml}$ of nicotinamide adenine dinucleotide (NAD; Germany) at $37^{\circ} \mathrm{C}$ with shaking at $180 \mathrm{rpm}$.

\section{Growth Curves of APPs}

To determine the optimal time point for harvesting Apx exotoxins, the growth curves of APP serotypes 1,2, and 5 in Mycoplasma Broth Base (PPLO; Oxoid Ltd., UK) or Columbia Broth (CB; Difco BD, USA) medium supplemented with $10 \mu \mathrm{g} / \mathrm{ml}$ of NAD were determined within $8 \mathrm{~h}$ of inoculation. Optical density at $600 \mathrm{~nm}$ $\left(\mathrm{OD}_{600 \mathrm{~nm}}\right)$ was used to measure the density of cultures hourly.

\section{Optimization of Medium Used for Apx Toxin Production}

To optimize the culture medium used for the production of a higher concentration of Apx toxins, PPLO or CB medium supplemented with $10 \mu \mathrm{g} / \mathrm{ml}$ of NAD was used to culture APP serotypes 1,2 , and 5 . The cultures were stopped after $6 \mathrm{~h}$ of cultivation at $37^{\circ} \mathrm{C}$ and $5 \% \mathrm{CO}_{2}$, and Apx toxins were harvested using the precipitation method, as described below.

\section{Optimization of $\mathrm{CaCl}_{2}$ Concentration for Apx Toxin Production}

In previous studies, calcium chloride $\left(\mathrm{CaCl}_{2}\right)$ was applied to increase the concentration of Apx toxins in APP serotype 1 and 5 cultures [14]. Here, we optimized the concentration of the $\mathrm{CaCl}_{2}$ supplement in APP serotype 2 cultures. A gradient of concentration ranging from 0 to $50 \mathrm{mM} \mathrm{CaCl}_{2}$ (Duchefa Biochemie B.V., The Netherlands) was applied to the cultures and the amount of Apx toxins was screened using SDS-PAGE.

\section{Collection of Apx Toxins Using the Precipitation Method}

Apx exotoxins were prepared from APP cultures according to the methods reported previously $[15,16]$. Cells were grown to the mid-log phase in the selected media containing $10 \mu \mathrm{g} / \mathrm{ml}$ of NAD and $20 \mathrm{mM} \mathrm{CaCl}$. The culture supernatants were collected using centrifugation at $8,000 \times g$ for $30 \mathrm{~min}$ at $4^{\circ} \mathrm{C}$ and mixed with $55 \%$ ammonium sulfate (Duchefa Biochemie B.V.), followed by incubation at $4^{\circ} \mathrm{C}$ overnight. The precipitated toxins were pelleted at $8,000 \times \mathrm{g}$ for $30 \mathrm{~min}$ at $4^{\circ} \mathrm{C}$, dissolved in $10 \mathrm{mM}$ Tris- $\mathrm{HCl}(\mathrm{pH} 7.5)$ [16] at a ratio of $1 / 85$ of the original culture volume, and dialyzed in the same buffer at $4^{\circ} \mathrm{C}$ overnight. The collected toxins were stored at $-70^{\circ} \mathrm{C}$ until use.

\section{Collection of Apx Toxins Using the Ultrafiltration Method}

To compare the efficacy of the precipitation and ultrafiltration methods of harvesting Apx toxins from APP serotype 2 cultures, the APP serotype 2 was inoculated in PPLO medium supplemented with $10 \mu \mathrm{g} / \mathrm{ml}$ of NAD and $20 \mathrm{~m} \mathrm{M} \mathrm{CaCl}_{2}$ for $6 \mathrm{~h}$ at $37^{\circ} \mathrm{C}$ with shaking at $180 \mathrm{rpm}$. For precipitation, Apx toxins were collected as described above. For ultrafiltration, the culture supernatants were collected using centrifugation at $8,000 \times g$ for $30 \mathrm{~min}$ at $4^{\circ} \mathrm{C}$, and Apx toxins were concentrated 85 -fold by ultrafiltration using Amicon Ultra-15 Centrifugal Filters (Merck, Ireland) at $5,000 \times g$ for $10 \mathrm{~min}$ at $4^{\circ} \mathrm{C}$ and stored at $-70^{\circ} \mathrm{C}$ until use. 

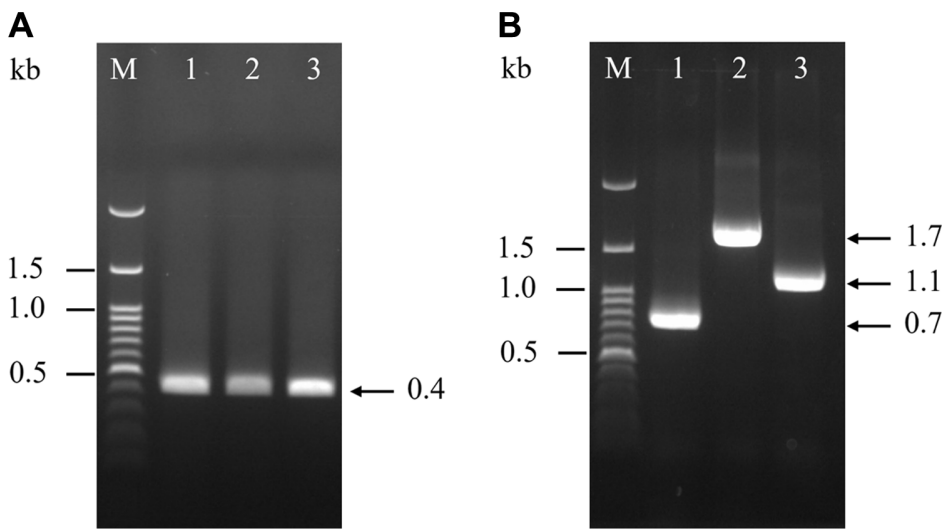

Fig. 1. PCR for identifying (A) and serotyping (B) APP serotypes 1, 2, and 5. M, marker; lane 1, APP serotype 1; lane 2, APP serotype 2; and lane 3, APP serotype 5.

\section{SDS-PAGE}

To evaluate the expression ability and purity of Apx toxins, the collected toxins were separated by $12 \%$ SDSPAGE and stained with Coomassie Brilliant Blue R-250 (Bio-Rad Laboratories Ltd., UK). To measure the concentration of the produced Apx toxins, the intensity of the target bands was compared with the intensity of the bands corresponding to the bovine serum albumin (BSA) standards (Thermo Fisher Scientific, USA) that were loaded onto the same gel, and concentrations were estimated based on the computerized arbitrary units [17] using the Gel Doc XR+ Imaging System (Bio-Rad Laboratories, USA).

\section{Statistical Analysis}

Statistical analyses were performed and graphs were generated using the GraphPad Prism 5 program. Statistically significant differences are indicated as follows: ${ }^{\star} p<0.05,{ }^{\star *} p<0.01$, and ${ }^{\star * \star} p<0.001$.

\section{Results}

\section{Identification and Serotyping of APPs}

The APP serotypes were determined according to the criteria described in previous reports [10-13]. First, the APP isolates were confirmed as a band of $0.4 \mathrm{~kb}$ by nested PCR (Fig. 1A), followed by serotyping as APP serotypes 1,2 , and 5 corresponding to bands of $0.7,1.7$, and $1.1 \mathrm{~kb}$, respectively (Fig. $1 \mathrm{~B}$ ).

Optimization of Medium Used for Production of Apx Toxins

In previous studies, CB was used for cultivating and producing Apx toxins from APP serotypes 1, 2, and 5 [16, $18,19]$. In this study, we compared the production of Apx toxins in APP cultures using PPLO and CB media.

To determine the optimal time point for harvesting Apx toxins from APP cultures, growth curves of APP serotypes 1, 2, and 5 in PPLO or CB medium containing $10 \mu \mathrm{g} / \mathrm{ml}$ of NAD were prepared. APP cultures reached the stationary phase at $6 \mathrm{~h}$ of inoculation for all three APP serotypes (Fig. 2). Therefore, this time point was chosen for harvesting Apx toxins in subsequent experiments.

As shown in Fig. 3, the PPLO medium supplemented with $10 \mu \mathrm{g} / \mathrm{ml}$ of NAD induced the production of a higher level of Apx toxins compared with the $\mathrm{CB}$ medium containing $10 \mu \mathrm{g} / \mathrm{ml}$ of NAD. For APP serotype 1, the concentration of Apx toxins harvested from the culture using the PPLO medium was significantly higher than that

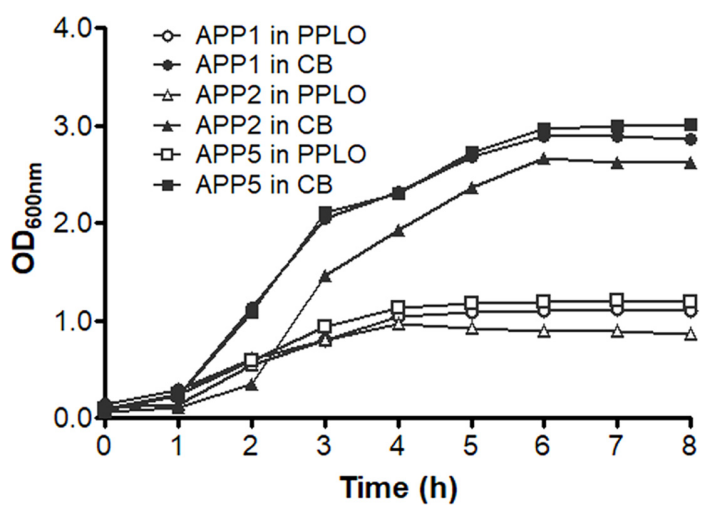

Fig. 2. Growth curves of APP serotypes 1, 2, and 5 in PPLO and CB media containing $10 \mu \mathrm{g} / \mathrm{ml}$ NAD within $8 \mathrm{~h}$ of cultivation. 
A

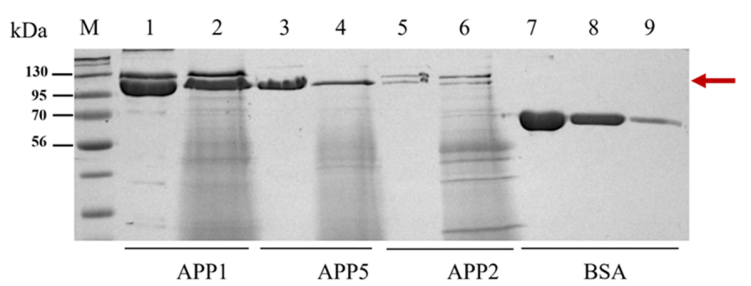

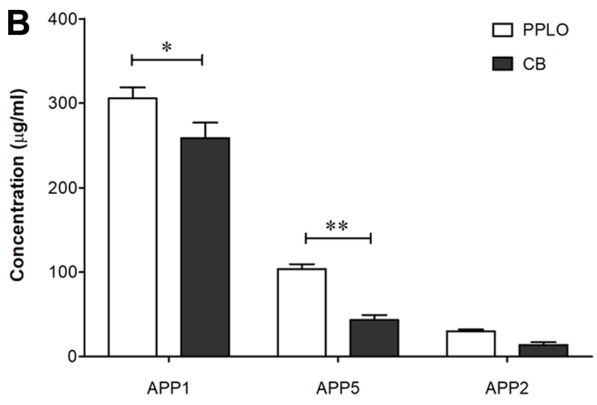

Fig. 3. Optimization of the medium used for the production of Apx toxins in APP serotype 1, 2, and 5 cultures. (A) SDS-PAGE analysis of the Apx toxins secreted from APP serotypes 1, 2, and 5 in PPLO and CB media containing $10 \mu \mathrm{g} / \mathrm{ml}$ NAD. M, protein marker; lanes 1, 3, and 5, Apx toxins in the PPLO medium; lanes 2, 4, and 6, Apx toxins in the CB medium; lanes 7, 8, 9, BSA standards (250, 125, and $25 \mu \mathrm{g} / \mathrm{ml}$, respectively). (B) The relative intensity of the SDS-PAGE bands illustrates the Apx toxin production yields. The asterisks indicate significant differences $\left({ }^{*} p<0.05 ;{ }^{*} p<0.01\right)$.

using the $\mathrm{CB}$ medium (mean, 305 and $258 \mu \mathrm{g} / \mathrm{ml}$, respectively; $p<0.05$ ) (Fig. 3B). In addition, the levels of Apx toxins collected from cultures of APP serotypes 2 and 5 were doubled in the PPLO medium compared with the CB medium (Fig. 3B), indicating that the PPLO medium had a positive effect on the production of Apx toxins. Furthermore, the Apx toxins produced by the three APP serotypes in the PPLO medium yielded a lower number of nonspecific bands and a clearer background on SDS-PAGE (Fig. 3A). In contrast, Apx toxins recovered from APP serotypes 1,2, and 5 cultured in the CB medium exhibited numerous bands and a darker background. Collectively, these findings demonstrated that the PPLO medium supplemented with $10 \mu \mathrm{g} / \mathrm{ml}$ NAD is more appropriate for the production of Apx toxins from APP serotype 1,2, and 5 cultures.

\section{Optimization of $\mathrm{CaCl}_{2}$ Concentration for Production of Apx Toxins}

Van Den (1997), Haga et al. (2007), and Frey and Nicolet (1988) established the concentration of $\mathrm{CaCl}_{2}$ in APP serotype 1 and 5 cultures $[16,18,19]$. In this study, we optimized the concentration of $\mathrm{CaCl}_{2}$ in PPLO medium with $10 \mu \mathrm{g} / \mathrm{ml}$ of NAD to increase the amount of Apx toxins produced in APP serotype 2 cultures.

The results obtained showed that dose-dependent changes in the concentration of Apx toxins accompanied the variation in the concentrations of $\mathrm{CaCl}_{2}$ in the culture medium (Fig. 4). $\mathrm{CaCl}_{2}$ supplementation at 10 , 20, and 30 $\mathrm{mM}$ increased the levels of Apx toxins in the cultures. The production yields of Apx toxins were significantly higher in the medium containing $20 \mathrm{mM}(p<0.001)$ and $30 \mathrm{mM}(p<0.01) \mathrm{CaCl}_{2}$ than they were in other cultures and in cultures without $\mathrm{CaCl}_{2}$, and reached a peak $(160 \mu \mathrm{g} / \mathrm{ml})$ at $20 \mathrm{mM} \mathrm{CaCl}_{2}$. In contrast, higher concentrations of $\mathrm{CaCl}_{2}$ appeared to have a negative effect on the production yields. Concentrations of $\mathrm{CaCl}_{2}$ of 40 and $50 \mathrm{mM}$ inhibited the expression of Apx toxins dramatically compared with $20 \mathrm{mM} \mathrm{CaCl}_{2}(p<0.001)$, to a level that was lower than that observed in cultures performed in PPLO medium without $\mathrm{CaCl}_{2}$. Taken together, these results indicate that PPLO medium supplemented with $10 \mu \mathrm{g} / \mathrm{ml}$ of NAD and $20 \mathrm{mM} \mathrm{CaCl}_{2}$ stimulates effectively the secretion of Apx toxins from APP serotype 2.

\section{A}

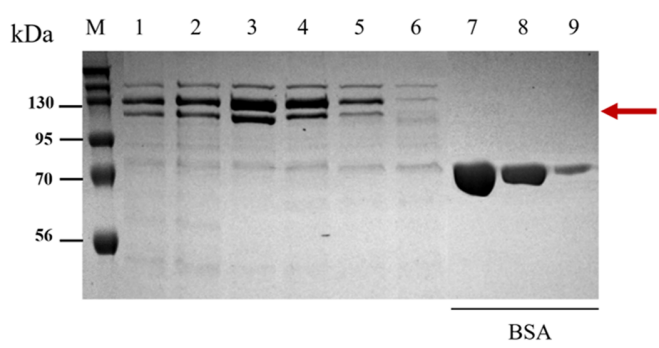

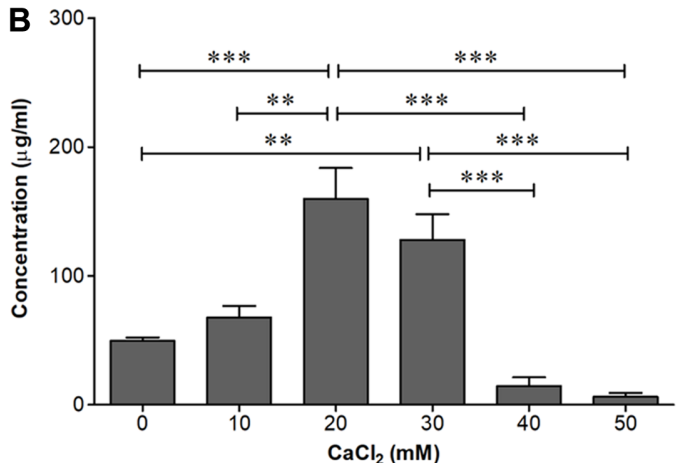

Fig. 4. Optimization of the $\mathrm{CaCl}_{2}$ concentration used for supplementation of APP serotype 2 cultures. (A) SDS-PAGE analysis of the Apx toxins secreted in APP serotype 2 cultures using PPLO medium containing $10 \mu \mathrm{g} / \mathrm{ml} \mathrm{NAD}$ and different concentrations of $\mathrm{CaCl}_{2}$. M, protein marker; lanes 1-6, the medium supplemented with 0-50 $\mathrm{mM} \mathrm{CaCl}_{2}$, respectively; lanes $7,8,9$, BSA standards $(250,125$, and $25 \mu \mathrm{g} / \mathrm{ml}$, respectively). (B) The relative intensity of the SDS-PAGE bands illustrates the Apx toxin production yields. The asterisks indicate significant differences $\left({ }^{* *} p<0.01 ;{ }^{* * *} p<0.001\right)$. 
A

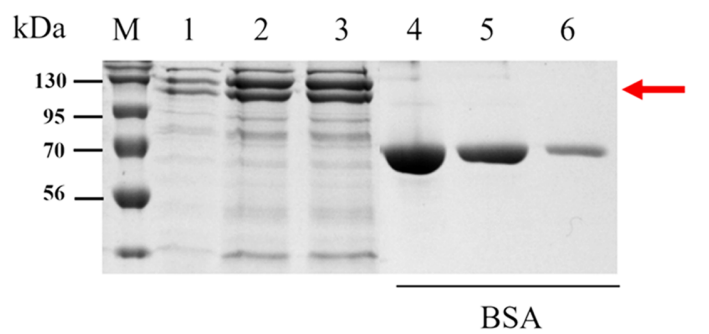

B

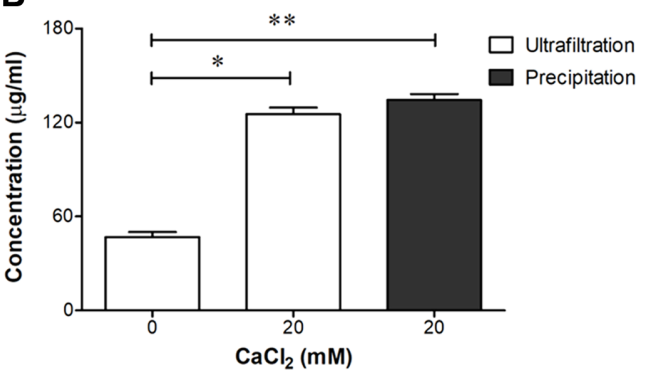

Fig. 5. Optimization of the harvesting method of Apx toxins in APP serotype 2 cultures. (A) SDS-PAGE analysis of the concentration of Apx toxins using different harvesting methods. M, protein marker; lane 1, PPLO medium containing $10 \mu \mathrm{g} / \mathrm{ml} \mathrm{NAD}$ without $\mathrm{CaCl}_{2}$, ultrafiltration method; lane 2, PPLO medium containing $10 \mu \mathrm{g} / \mathrm{ml} \mathrm{NAD} \mathrm{and} 20 \mathrm{mM} \mathrm{CaCl}_{2}$, ultrafiltration method; lane 3, PPLO medium containing $10 \mu \mathrm{g} / \mathrm{ml} \mathrm{NAD}$ and $20 \mathrm{mM} \mathrm{CaCl}_{2}$, precipitation method; lanes 4, 5, 6, BSA standards $(250,125$, and $25 \mu \mathrm{g} / \mathrm{ml}$, respectively). (B) The relative intensity of the SDS-PAGE bands illustrates the Apx toxin production yields. The asterisks indicate significant differences $\left({ }^{\star} p<0.05 ;{ }^{\star *} p<0.01\right)$.

\section{Selection of a Collection Method for Apx Toxins}

The concentration of $\mathrm{CaCl}_{2}$ was optimized to $20 \mathrm{mM}$, as it produced the highest level of Apx toxins. We compared the efficacy of the ultrafiltration and precipitation methods for harvesting Apx toxins in APP serotype 2 cultures. As shown in Fig. 5, the concentration of Apx toxins that were collected using the precipitation method was slightly higher than that obtained using the ultrafiltration method (mean, 134 and $125 \mu \mathrm{g} / \mathrm{ml}$, respectively); however, this difference was not significant $(p>0.05)$. This result suggests that the two purification methods can be applied to the collection of Apx toxins secreted from APP serotype 2 .

\section{Discussion}

APP serotypes 1 and 5 are predominant in Korea [20]. They are the most virulent serotypes and produce both ApxI and ApxII toxins [21, 22]. Moreover, the less virulent APP serotype 2, which produces ApxII and ApxIII toxins, also contributes to the infection of pigs in Korea [20]. Because of their prevalence and Apx toxin production patterns, these serotypes are used widely in the development of bacterins and Apx toxoid vaccines in Korea. Therefore, we optimized the culture conditions to improve the production yields of Apx toxins in APP serotype 1,2 , and 5 cultures.

In previous studies, CB, PPLO, tryptic soy broth (TSB), and brain heart infusion (BHI) media have been used for the cultivation of APP $[18,19,23-25]$. However, TSB and BHI media cause precipitation when supplemented with $20 \mathrm{mM} \mathrm{CaCl}_{2}$ for the enhancement of Apx toxin production. In contrast, $\mathrm{CB}$ and PPLO media do not cause precipitation after the addition of $\mathrm{CaCl}_{2}$; hence, they are suitable for Apx toxin production.

In this study, we described the process used to harvest high concentrations of Apx toxins from APP serotype 1, 2 , and 5 cultures. The growth curves of APP serotypes 1,2, and 5 in PPLO or CB media containing $10 \mu \mathrm{g} / \mathrm{ml}$ of NAD were determined (Fig. 2). It has been reported that the amount of Apx toxins varies throughout the APP growth curve, and peaks at a high APP cell density (from the late logarithmic to the early stationary phase) [26]. Therefore, the harvesting time was set at $6 \mathrm{~h}$ of inoculation in this study, which was consistent with previous studies [15, 16, 18, 25, 27].

SDS-PAGE using BSA as a standard is a suitable method for the quantification of specific protein bands of interest, for the determination of the purity of proteins and standardization of concentrations among different toxin preparations $[28,29]$. Hence, this method was used in several previous reports $[17,28-30]$. The target bands were confirmed as Apx toxins according to our previous study [31], in which the secretion genes were deleted, leading to the absence of the Apx toxin bands on SDS-PAGE. Frey and Nicolet (1988) also showed that precipitation using ammonium sulfate can separate the Apx toxins from several proteins [19]; thus, Apx toxins are predominant among the harvested proteins. These findings prove that the target bands exhibiting a molecular mass of $110-130 \mathrm{kDa}$ on SDS-PAGE are Apx toxins.

Although numerous studies have used the $\mathrm{CB}$ medium to produce Apx toxins [15, 16, 18, 19, 32], the optimization of production of these toxins using the PPLO medium has not been reported. Here, CB and PPLO media with equally added $10 \mu \mathrm{g} / \mathrm{ml}$ of NAD were applied to the production of Apx toxins in APP serotype 1,2, and 5 cultures (Figs. 3A and 3B). The levels of Apx toxins were significantly higher in the PPLO than those in the CB medium (Fig. 3B). The concentrations of Apx toxins obtained using the PPLO medium in this study were approximately 30,100 , and $300 \mu \mathrm{g} / \mathrm{ml}$ for APP serotypes 2, 5, and 1, respectively. In addition, the collected toxins yielded a clearer background on SDS-PAGE in the case of the PPLO medium (Fig. 3A). This result indicates that PPLO supplemented with $10 \mu \mathrm{g} / \mathrm{ml}$ of NAD is more appropriate for the production of Apx toxins from APP cultures, as it was more effective in the purification step and yielded fewer nonspecific components in the final products.

The Apx toxins contain glycine-rich nonapeptides that exhibit strong binding to $\mathrm{Ca}^{2+}$ cations [33]. Thus, $\mathrm{Ca}^{2+}$ plays an important role in the induction of the expression of Apx toxins in APP [14]. Supplementation with $\mathrm{CaCl}_{2}$ 
has been used to enhance the production of Apx toxins in the cultures of APP serotypes 1 and $5[16,18,19,26]$. Here, we optimized $\mathrm{CaCl}_{2}$ supplementation in APP serotype 2 cultures. To our knowledge, this is the first investigation of the increase in the levels of Apx toxins in APP serotype 2 cultures afforded by $\mathrm{CaCl}_{2}$ supplementation. After $6 \mathrm{~h}$ of cultivation of APP serotype 2 in PPLO medium containing $10 \mu \mathrm{g} / \mathrm{ml}$ of NAD and a range of $\mathrm{CaCl}_{2}$ concentrations, the toxins were collected using the precipitation method and analyzed. The addition of $\mathrm{CaCl}_{2}$ significantly increased the production yields of Apx toxins in APP serotype 2 cultures. The highest toxin concentration $(160 \mu \mathrm{g} / \mathrm{ml})$ was observed after supplementation with $20 \mathrm{mM} \mathrm{CaCl}_{2}$. In contrast, a reversion was observed in the presence of supplementation with higher concentrations ( 40 and $50 \mathrm{mM})$ of $\mathrm{CaCl}_{2}$, at which the production of the toxins in APP serotype 2 cultures was even decreased (Figs. 4A and 4B). Our results are consistent with those of previous studies, in which $\mathrm{CaCl}_{2}$ concentrations of 10 to $25 \mathrm{mM}$ were recommended for APP serotype 1 and 5 culture $[15,16,18,19]$. This result suggests that PPLO medium containing $10 \mu \mathrm{g} / \mathrm{ml}$ of NAD and $20 \mathrm{mM} \mathrm{CaCl}_{2}$ effectively stimulates the expression of Apx toxins in APP serotype 2 cultures.

Ultrafiltration and precipitation have been applied for harvesting Apx toxins from APP cultures. We conducted the first comparison between the two methods in the context of Apx toxin production from APP serotype 2 cultures. A slightly higher level of Apx toxins was observed using the precipitation method in comparison with the ultrafiltration method; however, this difference was not significant (Figs. 5A and 5B). Collectively, the results of the present study suggest a significant improvement in the levels of Apx toxins collected from APP cultures using an optimized medium.

In conclusion, in this study, we described the optimization of the culture medium and conditions, and the toxin collection method to enhance the yield of Apx toxins in APP serotype 1,2, and 5 cultures. In particular, the levels of Apx toxins in APP serotype 2 cultures were significantly increased using an optimized medium with an appropriate $\mathrm{CaCl}_{2}$ concentration and harvesting method. These findings may represent a good strategy for the development of toxoid vaccines or effective combination vaccines against APP infection.

\section{Acknowledgments}

This work was supported by the Technology Development Program to Innovac Co. S2540568, and funded by the Ministry of SMEs and Startups (MSS, Korea).

\section{Conflict of Interest}

The authors have no financial conflicts of interest to declare.

\section{References}

1. Sassu EL, Bossé JT, Tobias TJ, Gottschalk M, Langford PR, Hennig-Pauka I. 2018. Update on Actinobacillus pleuropneumoniaeknowledge, gaps and challenges. Transbound. Emerg. Dis. 65 Suppl 1: 72-90.

2. Liu J, Chen X, Lin L, Tan C, Chen Y, Guo Y, et al. 2007. Potential use an Actinobacillus pleuropneumoniae double mutant strain $\triangle a p x I I C \triangle a p x I V A$ as live vaccine that allows serological differentiation between vaccinated and infected animals. Vaccine 25: $7696-$ 7705.

3. Mei L, Zhou R, Lu HS, Bei WC, Liu WH, Lin LW, et al. 2006. Study on the immunogenicity of N-terminal polypeptide of RTX toxin I of Actinobacillus pleuropneumoniae. Chin. J. Biotechnol. 22: 39-45.

4. Bossé JT, Li Y, Sárközi R, Fodor L, Lacouture S, Gottschalk M, et al. 2018. Proposal of serovars 17 and 18 of Actinobacillus pleuropneumoniae based on serological and genotypic analysis. Vet. Microbiol. 217 : 1-6.

5. Ramjeet M, Deslandes V, Gouré J, Jacques M. 2008. Actinobacillus pleuropneumoniae vaccines: from bacterins to new insights into vaccination strategies. Anim. Health. Res. Rev. 9: 25-45.

6. Frey J. 1995. Virulence in Actinobacillus pleuropneumoniae and RTX toxins. Trends Microbiol. 3: 257-261.

7. Bossé JT, Janson H, Sheehan BJ, Beddek AJ, Rycroft AN, Kroll JS, et al. 2002. Actinobacillus pleuropneumoniae: pathobiology and pathogenesis of infection. Microbes Infect. 4: 225-235.

8. Chiers K, De Waele T, Pasmans F, Ducatelle R, Haesebrouck F. 2010. Virulence factors of Actinobacillus pleuropneumoniae involved in colonization, persistence and induction of lesions in its porcine host. Vet. Res. 41: 65.

9. Del Pozo Sacristán R, Michiels A, Martens M, Haesebrouck F, Maes D. 2014. Efficacy of vaccination against Actinobacillus pleuropneumoniae in two Belgian farrow-to-finish pig herds with a history of chronic pleurisy. Vet. Rec. 174: 302.

10. Schaller A, Djordjevic SP, Eamens GJ, Forbes WA, Kuhn R, Kuhnert P, et al. 2001. Identification and detection of Actinobacillus pleuropneumoniae by PCR based on the gene apxIVA. Vet. Microbiol. 79: 47-62.

11. Schuchert JA, Inzana TJ, Angen O, Jessing S. 2004. Detection and identification of Actinobacillus pleuropneumoniae serotypes 1, 2, and 8 by multiplex PCR. J. Clin. Microbiol. 42: 4344-4348.

12. Angen O, Ahrens P, Jessing SG. 2008. Development of a multiplex PCR test for identification of Actinobacillus pleuropneumoniae serovars 1, 7, and 12. Vet. Microbiol. 132: 312-318.

13. Lo TM, Ward CK, Inzana TJ. 1998. Detection and identification of Actinobacillus pleuropneumoniae serotype 5 by multiplex PCR. J. Clin. Microbiol. 36: 1704-1710.

14. Frey J, Nicolet J. 1988. Regulation of hemolysin expression in Actinobacillus pleuropneumoniae serotype 1 by Ca2+. Infect. Immun. 56: $2570-2575$.

15. Nielsen R, van den Bosch JF, Plambeck T, Sorensen V, Nielsen JP. 2000. Evaluation of an indirect enzyme-linked immunosorbent assay (ELISA) for detection of antibodies to the Apx toxins of Actinobacillus pleuropneumoniae. Vet. Microbiol. 71: 81-87.

16. Van Den Bosch JF (Boxmeer, NL). 1997. Actinobacillus pleuropneumoniae subunit vaccine. United States patent application 5648081

17. Wu HC, Yeh PH, Hsueh KJ, Yang WJ, Chu CY. 2018. Recombinant ApxIV protein enhances protective efficacy against Actinobacillus pleuropneumoniae in mice and pigs. J. Appl. Microbiol. 124: 1366-1376.

18. Haga Y, Ogino S, Ohashi S, Ajito T, Hashimoto K, Sawada T. 1997. Protective efficacy of an affinity-purified hemolysin vaccine against experimental swine pleuropneumonia. J. Vet. Med. Sci. 59: 115-120.

19. Frey J, Nicolet J. 1988. Purification and partial characterization of a hemolysin produced by Actinobacillus pleuropneumoniae type strain 4074. FEMS Microbiol. Lett. 55: 41-45.

20. Lee KE, Choi HW, Kim HH, Song JY, Yang DK. 2015. Prevalence and characterization of Actinobacillus pleuropneumoniae isolated from Korean pigs. J. Bacteriol. Virol. 45: 19-25. 
21. Komal JP, Mittal KR. 1990. Grouping of Actinobacillus pleuropneumoniae strains of serotypes 1 through 12 on the basis of their virulence in mice. Vet. Microbiol. 25: 229-240.

22. Yuan F, Liu J, Guo Y, Tan C, Fu S, Zhao J, et al. 2011. Influences of ORF1 on the virulence and immunogenicity of Actinobacillus pleuropneumoniae. Curr. Microbiol. 63: 574-580.

23. Šatrán P, Nedbalcová K, Kučerová Z. 2003. Comparison of protection efficacy of toxoid and whole-cell vaccines against porcine pleuropneumonia caused by endotracheal infection with Actinobacillus pleuropneumoniae. Acta Vet. Brno 72: 213-219.

24. Liao CW, Chiou HY, Yeh KS, Chen JR, Weng CN. 2003. Oral immunization using formalin-inactivated Actinobacillus pleuropneumoniae antigens entrapped in microspheres with aqueous dispersion polymers prepared using a co-spray drying process. Prev. Vet. Med. 61: 1-15.

25. Huter V, Hensel A, Brand E, Lubitz W. 2000. Improved protection against lung colonization by Actinobacillus pleuropneumoniae ghosts: characterization of a genetically inactivated vaccine. J. Biotechnol. 83: 161-172.

26. Jarma E, Regassa LB. 2004. Growth phase mediated regulation of the Actinobacillus pleuropneumoniae ApxI and ApxII toxins. Microb. Pathog. 36: 197-203.

27. Hensel A, Stockhofe-Zurwieden N, Petzoldt K, Lubitz W. 1995. Oral immunization of pigs with viable or inactivated Actinobacillus pleuropneumoniae serotype 9 induces pulmonary and systemic antibodies and protects against homologous aerosol challenge. Infect. Immun. 63: 3048-3053.

28. Crespo AL, Spencer TA, Nekl E, Pusztai-Carey M, Moar WJ, Siegfried BD. 2008. Comparison and validation of methods to quantify CrylAb toxin from Bacillus thuringiensis for standardization of insect bioassays. Appl. Environ. Microbiol. 74: 130-135.

29. Knight MI, Chambers PJ. 2003. Problems associated with determining protein concentration: a comparison of techniques for protein estimations. Mol. Biotechnol. 23: 19-28.

30. Phonvisay M, Lee JW, Liou JJ, Wang HY, Chu CY. 2019. Evaluation of long-term antibody response and cross-serotype reaction in ducks immunised with recombinant Riemerella anatipestifer outer membrane protein A and CpG ODN. J. Vet. Res. 63: 543-548.

31. Dao HT, Truong QL, Do VT, Hahn TW. 2020. Construction and immunization with double mutant $\triangle a p x I B D \triangle p n p$ forms of Actinobacillus pleuropneumoniae serotypes 1 and 5. J. Vet. Sci. 21: e20.

32. Lin L, Bei W, Sha Y, Liu J, Guo Y, Liu W, et al. 2007. Construction and immunogencity of a $\Delta a p x I C / \Delta a p x I I C$ double mutant of Actinobacillus pleuropneumoniae serovar 1. FEMS Microbiol. Lett. 274: 55-62.

33. Frey J. 2011. The role of RTX toxins in host specificity of animal pathogenic Pasteurellaceae. Vet. Microbiol. 153: 51-58. 